\title{
Olfactory subsystems in the honeybee: sensory supply and sex specificity
}

\author{
Jan Kropf • Christina Kelber • Kathrin Bieringer • \\ Wolfgang Rössler
}

Received: 7 February 2014 / Accepted: 8 April 2014 /Published online: 13 May 2014

(C) The Author(s) 2014. This article is published with open access at Springerlink.com

\begin{abstract}
The antennae of honeybee (Apis mellifera) workers and drones differ in various aspects. One striking difference is the presence of Sensilla basiconica in (female) workers and their absence in (male) drones. We investigate the axonal projection patterns of olfactory receptor neurons (ORNs) housed in S. basiconica in honeybee workers by using selective anterograde labeling with fluorescent tracers and confocalmicroscopy analysis of axonal projections in antennal lobe glomeruli. Axons of S. basiconica-associated ORNs preferentially projected into a specific glomerular cluster in the antennal lobe, namely the sensory input-tract three (T3) cluster. T3associated glomeruli had previously been shown to be innervated by uniglomerular projection (output) neurons of the medial antennal lobe tract (mALT). As the number of T3 glomeruli is reduced in drones, we wished to determine whether this was associated with the reduction of glomeruli innervated by medial-tract projection neurons. We retrogradely traced mALT projection neurons in drones and counted the innervated glomeruli. The number of mALT-associated glomeruli was strongly reduced in drones compared with workers. The preferential projections of $S$. basiconica-associated ORNs in T3
\end{abstract}

This work was supported by the DFG Priority Program SPP 1392 "Integrative Analysis of Olfaction" (RO 1177/5-2 to W.R.). C.K. is funded by the Deutsche Forschungsgesellschaft (DFG KE 1701/1).

Electronic supplementary material The online version of this article (doi:10.1007/s00441-014-1892-y) contains supplementary material, which is available to authorized users.

J. Kropf $(\bowtie) \cdot$ C. Kelber $\cdot$ K. Bieringer $\cdot$ W. Rössler

Department of Behavioral Physiology and Sociobiology,

Biozentrum, University of Würzburg, Am Hubland,

97074 Würzburg, Germany

e-mail: jan.kropf@uni-wuerzburg.de

C. Kelber

Ecological Networks, Technical University of Darmstadt,

Schnittspahnstrasse 3, 64287 Darmstadt, Germany glomeruli together with the reduction of mALT-associated glomeruli support the presence of a female (worker)-specific olfactory subsystem that is partly innervated by ORNs from S. basiconica and is associated with the T3 cluster of glomeruli and mALT projection neurons. We propose that this olfactory subsystem supports parallel olfactory processing related to worker-specific olfactory tasks such as the coding of colony odors, colony pheromones and/or odorants associated with foraging on floral resources.

Keywords Sensilla basiconica · Antennal lobe · Glomeruli · Projection neuron $\cdot$ Honeybee drone

\section{Introduction}

Olfaction is an ancient sensory modality and plays a crucial role in most animals for approaching or avoiding various odor sources and for judging their quality in a variety of behavioral contexts. Whereas odorant reception at the molecular level exhibits distinct differences between vertebrates and insects, the basic wiring pattern of receptor neurons with second-order neurons within the primary olfactory centers, the vertebrate olfactory bulb and the insect antennal lobe (AL) shows several striking similarities. These have been the subject of intense research over recent years (for reviews, see Hildebrand and Shepherd 1997; Ache and Young 2005; Touhara and Vosshall 2009; Martin et al. 2011).

Insect antennae are covered with various types of sensory sensilla; most of them being specialized for chemoreception but also for hygro-, mechano- and thermoreception (Esslen and Kaissling 1976; Maronde 1991; Ai et al. 2007). Olfactory sensilla house the olfactory receptor neurons (ORNs) that extend axons into spheroidal structures termed glomeruli to form synaptic connections with local interneurons and projection neurons (PNs). Glomeruli represent the functional units 
of the AL (e.g., Anton and Homberg 1999). In the honeybee, Sensilla placodea, Sensilla trichoidea and Sensilla basiconica have been classified as olfactory sensilla, either according to their odor-response profiles in single-sensillum recordings (S. placodea) or based on specific anatomical features (S. trichoidea, S. basiconica; Lacher 1964; Esslen and Kaissling 1976; Akers and Getz 1993; Getz and Akers 1993). ORN axons from olfactory sensilla project via four distinct $\mathrm{AL}$ sensory-input tracts to four clusters of glomeruli in the $\mathrm{AL}$ termed the T1-T4 cluster (Mobbs 1982; Galizia et al. 1999; Abel et al. 2001; Kirschner et al. 2006). Axons from ORNs in $S$. placodea project to all four clusters of glomeruli T1-T4 (Brockmann and Brückner 1995; Kelber et al. 2006) and single-sensillum recordings from $S$. placodea have revealed responses to a broad range of odorants (Getz and Akers 1993). This might be either caused by the broad tuning of ORNs or attributable to the finding that $S$. placodea house many individual ORNs (between 7 and 23; Kelber et al. 2006), each covering a certain spectrum of molecular receptive ranges.

In insects, PNs convey the olfactory information to the mushroom bodies (MBs), higher sensory association centers and sites associated with learning and memory (Gerber et al. 2004; Davis 2005; Giurfa 2007; Hourcade et al. 2010; Cervantes-Sandoval et al. 2013). Uniglomerular PNs in the honeybee and other Hymenoptera have been shown to project to the MBs and lateral horn (LH) via two parallel tracts: the medial and the lateral AL tracts (mALT and 1ALT) forming a dual olfactory pathway (Abel et al. 2001; Kirschner et al. 2006; Galizia and Rössler 2010; Rössler and Brill 2013; tract terminology according to Ito et al. 2014). Comparative anatomical studies indicate that a dual olfactory pathway probably emerged in the basal Hymenoptera (Dacks and Nighorn 2011; Rössler and Zube 2011; Rössler and Brill 2013). However, the selective pressure that promoted the evolution of a dual olfactory pathway within this group of insects remains to be further investigated (Rössler and Brill 2013).

Female honeybee workers show complex social behavior that is largely influenced by pheromonal communication (Slessor et al. 2005; Le Conte and Hefetz 2008). This is different in male drones, which mainly perform reproductive tasks, do not forage actively for food and might not need to distinguish minor changes in colony pheromone concentrations. On the other hand, drones are highly sensitive to the queen sex-pheromone (Gary 1962). Therefore, differences in the olfactory system reflecting these behavioral specializations are likely to exist between honeybee workers and drones. One striking sex-specific difference is the absence of S. basiconica on drone antennae (Esslen and Kaissling 1976; Nishino et al. 2009). Furthermore, drone ALs contain a smaller number of glomeruli compared with both female castes (workers and queens) but comprise several enlarged macroglomeruli (Arnold et al. 1985; Sandoz 2006; Groh and Rössler 2008; Nishino et al. 2009). The largest macroglomerulus has been shown to respond to the major component of the queen mandibular pheromone (Sandoz 2006; Wanner et al. 2007). The reduction of AL glomeruli is mostly associated with the T3 cluster (Nishino et al. 2009), which has been demonstrated to be mainly innervated by medial tract PNs in honeybee workers (Kirschner et al. 2006). Comparative studies in other Hymenoptera indicate that the lack of $S$. basiconica in males is a characteristic trait across both social and solitary Hymenoptera (Ågren 1977, 1978; Wcislo 1995; Ågren and Hallberg 1996; Nakanishi et al. 2009, 2010; Mysore et al. 2010; Nishikawa et al. 2012; Streinzer et al. 2013). In the leaf-cutting ant Atta vollenweideri, S. basiconica have been found exclusively to innervate a specific (T6) cluster of AL glomeruli (Kelber et al. 2010).

The absence of $S$. basiconica, together with the reduction of glomeruli in the T3 cluster, in honeybee drones suggests that ORNs from S. basiconica preferentially innervate glomeruli in the T3 cluster and are associated with medial-tract PNs. To test this hypothesis, we investigated the axonal projections of ORNs in the hair-like olfactory antennal sensilla of female worker bees, with a special focus on $S$. basiconica and, in particular, their glomerular innervation patterns and their association with PN output tracts. Furthermore, we retrogradely labeled the mALT in drones to analyze whether glomeruli associated with this tract are reduced.

\section{Materials and methods}

\section{Animals}

Honeybee workers and drones (Apis mellifera carnica) were collected from bee hives of the institutional bee station at the University of Würzburg. Workers were taken from the entrance of the hive and drones were caught directly in the hive. In both cases we did not control for age. The animals were cooled in a refrigerator $\left(4^{\circ} \mathrm{C}\right)$, fixed in custom made plastic holders and provided with sugar solution $(40 \%)$ ad libitum.

\section{Staining of axonal projections}

ORNs from hair-like sensilla on defined antennal segments were labeled by using a method previously described by Kelber et al. (2010). Cut glass microelectrodes were placed close to a defined segment of the antenna under visual control $(280 \times)$ by using a photo-microscope (M 400, Wild, Heerbrugg, Germany) or an Olympus imaging system (200×-400×, upright microscope: BX51WI, filter set: UMF2 excitation 395/440 FT 460 emission 540/50, objective: XLUMP, NA 0.95, light source: MT20, software: Cell R v2.5 
[all Olympus Imaging Europa], camera: model 8484-03G [Hamamatsu Photonics]) and a micromanipulator (Junior Unit, Luigs \& Neumann, Ratingen, Germany). This setup allowed the identification of individual sensilla (Fig. 1a, b). The electrode was mounted on a piezo element (Element, EPZ-Serie, Conrad Electronic, Hirschau, Germany) connected to a function generator (PM 5133, Fluke/Philips, Kassel, Germany). Vibration $(2-13 \mathrm{kHz})$ of the piezo element and therefore also of the pipette was used to cut the hair-like sensilla and to expose the dendrites of the ORNs within sensilla of a defined antennal segment. Subsequently, a droplet of Biotin Dextran (Molecular Probes, D-7135, Leiden, Netherland) or Microruby (tetramethylrhodamine dextran with biotin, 3,000 MW, lysine-fixable, D-7162; Molecular Probes, Eugene, Ore., USA), 3-5\% in distilled water, was applied onto the remaining sensilla stumps to enable the dye to enter the ORN dendrites. This method allowed selective staining of hair-like sensilla (S. trichoidea, S. basiconica) and excluded the staining of $S$. placodea, as these sensilla are located beneath a plate-like structure of the antennal cuticle (Esslen and Kaissling 1976; Kelber et al. 2006). Animals were then kept in the dark for $24 \mathrm{~h}$ before the brains were dissected. For the staining of single sensilla, a microelectrode was placed close to one sensillum to cut only a single sensillum hair. Because of their small size, the cutting of individual basiconic sensilla was extremely difficult and the success rate for staining was extremely low (only one staining after the approved cut of only one $S$. basiconicum). Therefore, in most cases, we moved the electrode, in particular in S. basiconica-rich regions close to the borders of antennal segments, to cut small groups of hair-like sensilla preferentially including $S$. basiconica (Fig. 1a, b). Larger numbers of $S$. basiconica could be found in ring-like arrangements in the distal region of the antennal segments 3-10, close to the transition to the next segment (Fig. 1; Esslen and Kaissling 1976; Nishino et al. 2009). These axonal projections in this staining technique were compared with the staining of ORN axons from $S$. trichoidea only. Larger groups of $S$. trichoidea in the middle of antennal segments that lacked any S. basiconica (Fig. 1) were cut with a sharp tungsten wire (diameter: $200 \mu \mathrm{m}$ ) attached to the piezo element. Microelectrodes used in all experiments (including mALT staining; see below) were pulled from thin-walled glass pipettes (1B100F-3, WPI, Sarasota, USA) with a DMZ-Universal Puller (ZeitzInstruments, Martinsried, Germany).

\section{Staining of medial tract projection neurons in drones}

PNs of the mALT were retrogradely stained by using methods described in detail by Kirschner et al. (2006), Zube et al. (2008) and Rössler and Zube (2011). Head capsules were opened and glands and tracheae covering the brain were removed. The tissue between the vertical lobe of the MB and the AL was punctured with a fine glass electrode on the medial side, where mALT fibers run relatively close to the surface of the brain. A few crystals of either Microruby or Biotin Dextran were gently applied to the punctured tissue by means of a broken micropipette. Mass-staining of all AL output tracts was achieved by using a similar technique and application of dye crystals into the center of the AL. Massstaining of AL glomeruli was performed by the cutting of the antennae and the application of a droplet of Microruby (about 3-5\% in distilled water) to the antennal nerve stump. In all cases, animals were kept for $4-6 \mathrm{~h}$ in the dark under moist conditions to allow the dye to be transported intracellularly.

\section{Single PN staining}

For single PN staining, the glial sheath was gently removed from the AL with fine forceps. PN cell bodies were approached with a patch-clamp pipette under visual control by using an upright microscope (BX51WI, Olympus Imaging Europa) and a micromanipulator (Junior Unit, Luigs \& Neumann). We employed the intracellular [(K-gluconate (110 mM), HEPES (25 mM), KCl (10 mM), MgCl2 (5 mM), Mg-ATP (3 mM), Na-GTP (0.5 mM), EGTA (0.5 mM), pH 7.2, $284 \mathrm{mOsm})]$ and extracellular $[(\mathrm{NaCl}$ (140 mM), $\mathrm{KCl}(5 \mathrm{mM}), \mathrm{MgCl} 2$ (1 mM), CaCl2 (2.5 mM), NaHCO3 (4 mM), NaH2PO4 (1.2 mM), HEPES (6 mM), glucose (14 mM), pH 7.4, $326 \mathrm{mOsm})]$ solutions of Palmer
Fig. 1 Light microscopic images of Sensilla basiconica $(\mathrm{Sb})$, Sensilla placodea $(\mathrm{Sp})$ and Sensilla trichoidea $(S t ., S t)$ on a honeybee worker antenna. a Overview of the ninth segment. Bar $100 \mu \mathrm{m}$. b Detailed view of the $S$. basiconica-rich region on the ninth segment (white asterisks bases of two identifiable S. basiconica). Bar $25 \mu \mathrm{m}$
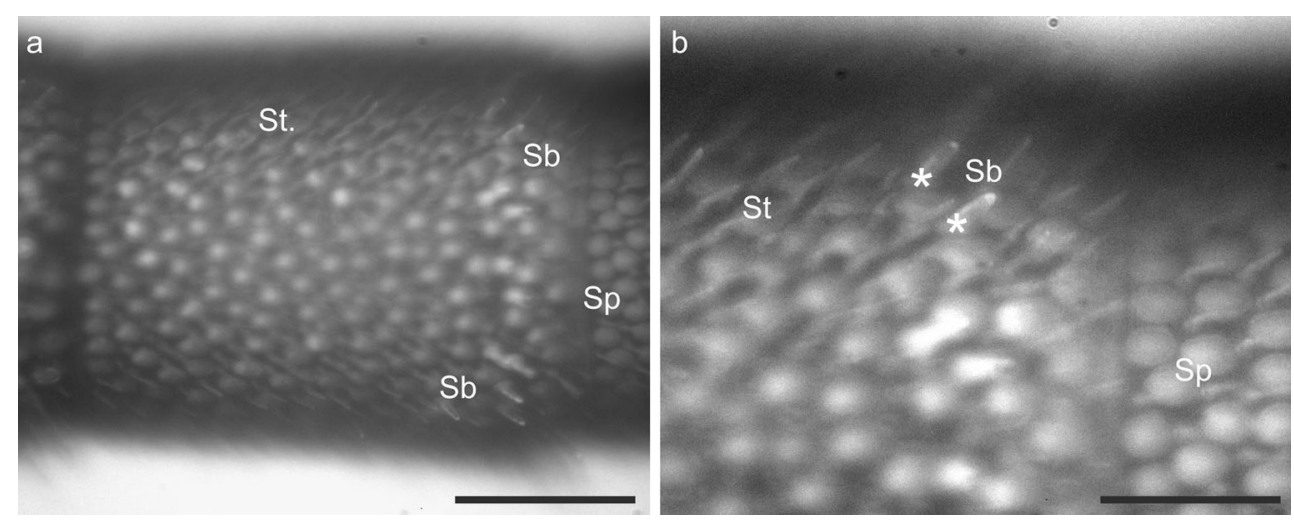
et al. (2013). For staining, 0.5-1 \% Lucifer Yellow (L0259, Sigma-Aldrich Chemie, Steinheim, Germany) was added to the intracellular solution. Whole cell patch clamp recordings were established by using an Axon system (Axopatch 200B, Digidata 1440A, ClampEx, Molecular Devices, Oregon, USA) and the membrane voltage was kept at $-60 \mathrm{mV}$.

\section{Neuranatomical analyses}

Brains were placed in fixative solution ( $4 \%$ formaldehyde) overnight, following the staining procedure and then rinsed five times for $10 \mathrm{~min}$ in PBS (phosphate-buffered saline, $\mathrm{pH}$ 7.2). Biotin-Dextran-injected brains were stained with Alexa488-conjugated Streptavidin (S-11226, Molecular Probes, Eugene, Ore., USA) in PBS with $0.2 \%$ Triton X (1:125) for $48 \mathrm{~h}$. Subsequently, brains were again rinsed five times for 10 min in PBS. Microruby- and Biotin-Dextran-labeled brains were dehydrated in an ascending ethanol series (50, 70, 90, $95,2 \times 100 \%$, each $10 \mathrm{~min}$ ) and cleared and mounted in methyl salicylate (M2047, Sigma-Aldrich Chemie) for confocal microscopy. Afterwards, brains were scanned with a confocal laser-scanning microscope (Leica TCS SP2; Leica Microsystems, Wetzlar, Germany). Images were processed with AMIRA 3.1.1 and 5.4 software (Mercury Computer Systems, Berlin, Germany) and AL glomeruli in one preparation were reconstructed by using the AMIRA wrapping tool. Image stacks were further processed with ImageJ 1.46j (Wayne Rasband, National Institutes of Health, Befesta, Md., USA). To count the glomeruli in all other successful mALT stainings (that were not three-dimensionally reconstructed in detail), we marked glomeruli in image stacks by using the segmentation editor implemented in ImageJ. Contrast and brightness were adjusted with GIMP 2.8.2 (GNU Image Manipulation Program, http://www.gimp.org) and images in figures were arranged with CorelDrawX6 (Corel, Ottawa, ON, Canada).

\section{Identification of glomeruli}

After the successful staining of axonal projections, AL reconstructions in workers were mapped onto a template AL by using the VOI (volume of interest) method described in Kelber et al. (2006). The antennal nerve, the T1 tract and a specific glomerulus (A17; after Galizia et al. 1999) were used as landmarks. Additionally, one AL reconstruction of a massstaining of ORN axons of the whole antennal nerve was mapped onto the same template AL. This reconstruction allowed the identification of the AL input tracts in the template AL. With the identified glomeruli and the input tracts in the template $\mathrm{AL}$, the matching of sensilla-stained ALs allowed the assignment of axonal projections of ORNs to a specific input tract and to particular glomeruli.

\section{Statistics}

Relative innervation frequencies of the input clusters (T1-T4) were analyzed with a Friedman analysis of variance (ANOVA) and a post-hoc Wilcoxon rank sum test with Bonferroni correction for multiple comparisons. The distribution of ORN axons in the various glomerular clusters originating from the staining of $S$. basiconica-rich regions was compared with a hypothetical distribution in the glomerular input clusters based on the natural distribution by using Fisher's exact test. All statistic tests were performed with R 2.10.1 (R Foundation for Statistical Computing, Vienna, Austria).

\section{Results}

ORN axon projections from many $S$. trichoidea and S. basiconica are broadly distributed into AL glomeruli

In the honeybee, ORN axons from S. placodea are broadly distributed across glomeruli of the AL and do not preferentially terminate in a specific sensory input cluster (Kelber et al. 2006). To determine whether this also held true for hair-like sensilla, we started with unselective mass-stainings of hairlike sensilla (S. trichoidea and S. basiconica) on a proximal (4th) and a distal (9th) segment of the same antenna by using two fluoresecent tracers. With this mass-staining method, no specific tract preferences of axons from ORNs housed in hairlike sensilla was found. However, the trajectories of axonal projections revealed a conspicuous axon-sorting-zone-like region at the $\mathrm{AL}$ entrance in which axons from the two stained axon bundles in the antennal nerve intermingled (Fig. 2a). Similarly, at the end of the antennal sensory input tracts, ORN axons showed a typical crossing pattern before they diverged to form terminal arborizations in specific subcompartments of individual glomeruli (Fig. 2a). Several glomeruli were innervated by ORN axons from the distal and the proximal segments. Interestingly, ORN axons from different antennal segments arborized in different layers within the same glomerulus (Fig. 2a, b). The dendritic arborizations of a single PN, in contrast to ORN axons, ramified across the entire volume of a glomerulus (Fig. 2b).

Axons from ORNs in S. trichoidea of a single antennal segment arborize in the T1, T2 and T3 cluster of glomeruli

As we found no specific preference of ORN projections for a certain glomerular cluster with unselective mass-staining of hair-like sensilla on different antennal segments, we set out to focus on the staining of ORNs from S. trichoidea only. Selective mass-labeling of axons from ORNs in S. trichoidea in honeybee workers was performed by cutting sensilla only at the middle region of the fifth antennal segment 


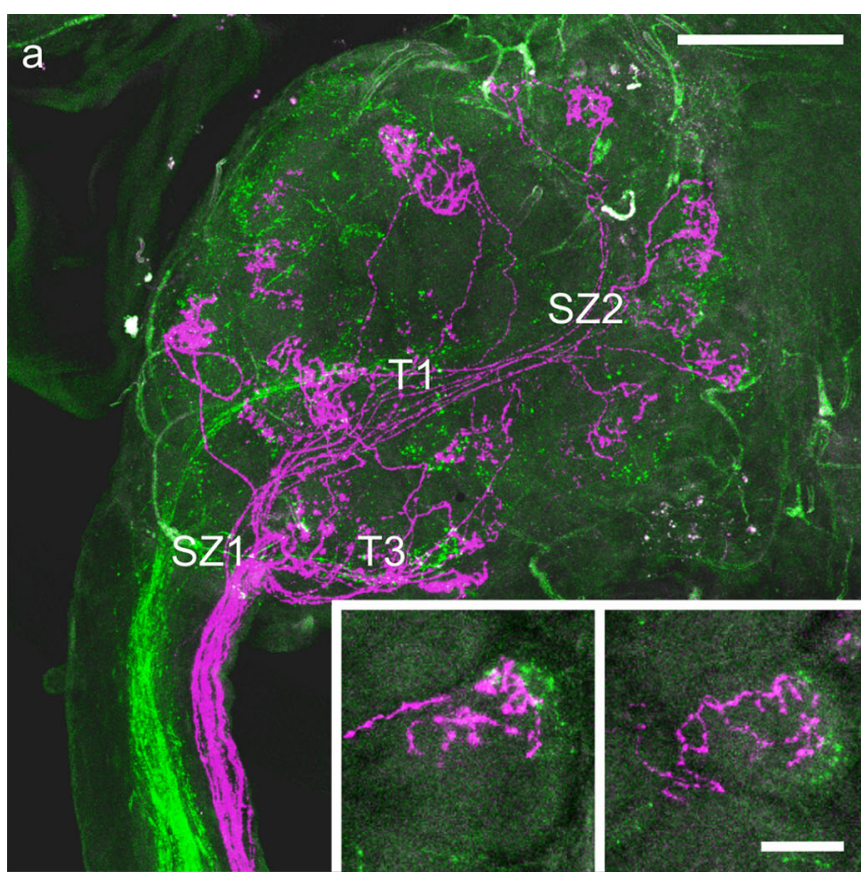

Fig. 2 a Z-projection of a double-mass-staining of hair-like sensilla on segment 9 (magenta) and segment 4 (green) of honey bee antenna. The input tracts $T 1$ and $T 3$ are indicated. Note the two sorting zones ( $S Z 1$, SZ2). Bar $100 \mu \mathrm{m}$. Insets Detailed views of two glomeruli that are innervated by axons from olfactory receptor neurons from both the distal

(Fig. 1). This mass-labeling technique excludes S. basiconia, which are located at the segment borders only but might well contain some mechanosensitive $S$. trichoidea (Lacher 1964) in addition to the olfactory sensilla. This staining $(n=13)$ revealed bundles of ORN axons terminating in the AL; a typical example is shown in Fig. 3. The trajectories of labeled ORN axons clearly changed from a more or less parallel organization into sorting-zone-like crossing patterns close to the entrance to the AL. From there, axonal projections proceeded into three tracts (T1, T2, T3) leading to the associated glomerular clusters of the AL. The T4 input tract and the associated glomerular cluster were not stained in any of the 13 preparations (Fig. 3d). A few axons proceeded into the dorsal lobe indicating that some of the stained sensilla stained were mechanosensory in nature (data not shown).

Axons from ORNs in S. basiconica preferentially innervate the $\mathrm{T} 3$ cluster of glomeruli

As $S$. basiconica are far less numerous compared with $S$. trichoidea, a preference of axons from ORNs housed in $S$. basiconica for a certain input cluster might be masked in the mass-staining of hair-like sensilla. We therefore selectively stained sensilla in S. basiconica-rich regions; this was successful in 15 worker bees. In all cases, axonal branches of stained ORN axons were mainly restricted to the outer regions of individual glomeruli (Fig. 4). Glomerular borders were visualized by autofluorescence. Between 3 and 29 glomeruli per bee were

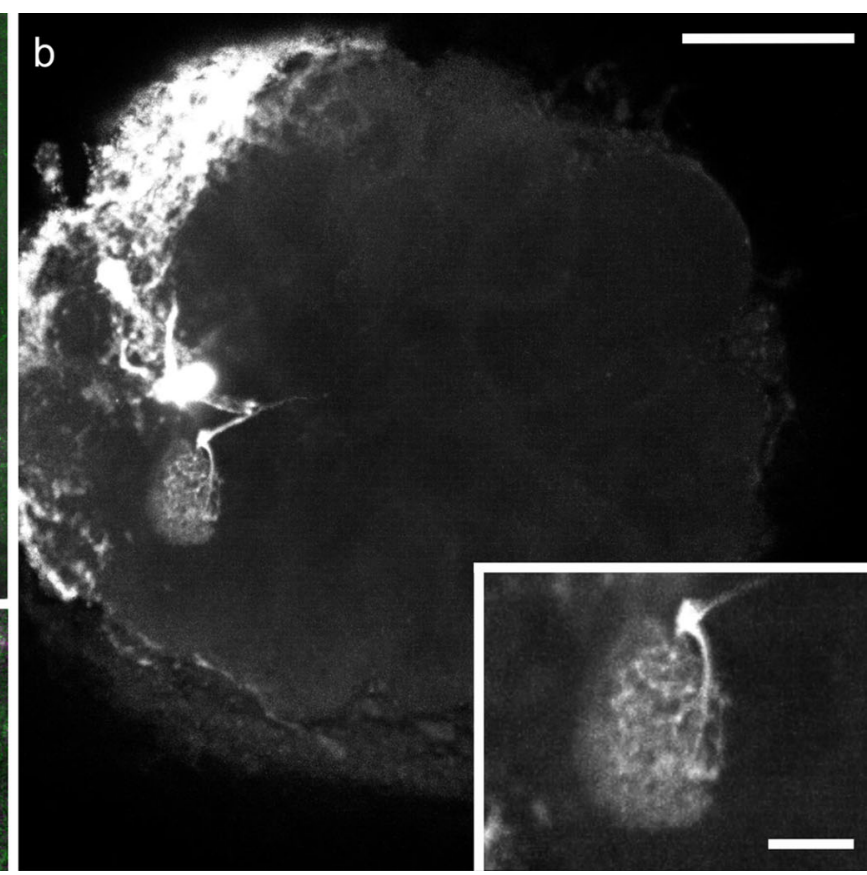

and the proximal parts of segment 9 in a layered fashion. Bar $25 \mu \mathrm{m}$. b Zprojection of an antennal lobe with an intracellularly stained projection neuron innervating a single glomerulus. Bar $100 \mu \mathrm{m}$. Inset Stained glomerulus in more detail; the dendritic arborizations of a projection neuron ramify throughout the entire glomerulus. Bar $25 \mu \mathrm{m}$

stained in the different preparations. All stained glomeruli were mapped into the template $\mathrm{AL}$ and assigned to their input-tract glomerular clusters. In 13 out of 15 bees, T3-associated glomeruli were the most frequently stained glomeruli. In total, 180 ORN axons were stained in 15 bees; 57 projected to T1, four to T2, 111 to T3 and eight to T4 (Fig. 5; exact numbers of stained glomeruli can be found in supplementary Table 1). In one case, we were able to determine, by visual inspection, that the staining was from only a single $S$. basiconicum that had been cut (Fig. 4g-i). In this case, a single glomerulus was labeled in the $\mathrm{T} 1$ cluster and nine glomeruli were innervated in the $\mathrm{T} 3$ cluster. As $S$. trichoidea were also present in the segmental border regions of the antennae enriched with $S$. basiconica, we could not completely exclude that some $S$. trichoidea were also stained in most of the other group stainings of sensilla in the S. basiconica-enriched regions. To test this statistically, we calculated the relative innervation frequencies for each specimen. We compared these frequencies with a Friedman ANOVA $\left(P=5.28^{*} 10^{-8}, \mathrm{chi}^{2}=36.72, n=15\right)$ and observed differences between at least two groups within the data. A post-hoc Wilcoxon rank sum test with Bonferroni correction for multiple comparisons revealed $\mathrm{T} 3$ as the most frequently stained cluster of glomeruli. Significant differences were found between all clusters, except between T2 and T4 (Fig. 5, exact $P$-values: T1:T2 $4.9 \times 10^{-4} \mathrm{~T} 1: \mathrm{T} 36.3 \times 10^{-4} \mathrm{~T} 1: \mathrm{T} 42.3 \times 10^{-3} \mathrm{~T} 2: \mathrm{T} 3$ $9.5 \times 10^{-6} \mathrm{~T} 2: \mathrm{T} 41.0 \mathrm{~T} 3: \mathrm{T} 41.5 \times 10^{-5}$ ). As the distribution of glomeruli among the input clusters is not homogenous (Flanagan and Mercer 1989; Galizia et al. 1999), we calculated 
Fig. 3 Images of a representative mass-staining of the axons of olfactory receptor neurons in Sensilla trichoidea of segment 5 of the honeybee worker antenna. Axons of olfactory receptor neurons are shown in magenta; the background was visualized via autofluorescence of the tissue and is shown in green. a Zprojection of $20 \mu \mathrm{m}$ of the dorsal part of the stained antennal lobe; the glomeruli innervated from T1 input tract (Tl) are clearly visible. b Z-projection of $20 \mu \mathrm{m}$ of the dorsal middle part of the stained antennal lobe. Glomeruli innervated from the $\mathrm{T} 3$ input tract (T3) and the T1 input tract (T1) are clearly visible. $\mathbf{c} Z$-projection of $20 \mu \mathrm{m}$ of the ventral middle part of the stained antennal lobe. Glomeruli innervated from the T3 input tract (T3) and the T2 input tract (T2) are clearly visible. d Zprojection of $20 \mu \mathrm{m}$ of the ventral part of the stained antennal lobe. Glomeruli innervated from the $\mathrm{T} 3$ input tract (T3) are clearly visible; the only non-innervated glomeruli (probably associated with T4 input tract) are indicated by the dashed circle. Bar $200 \mu \mathrm{m}$
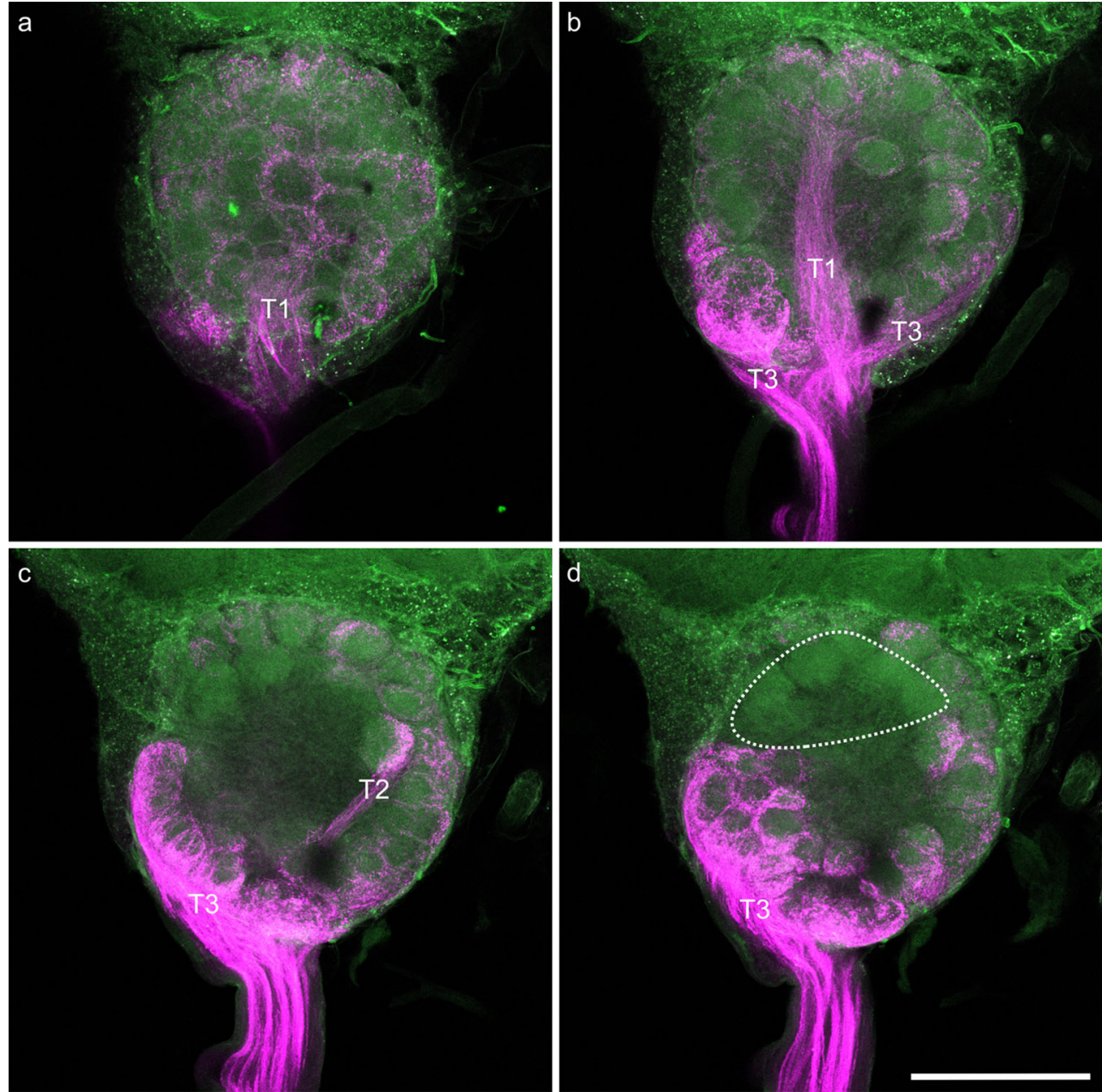

a hypothetical distribution of glomeruli (T1: 79, T2: 8, T3: 85 , T4: 8) by using the total number of glomeruli (180) stained in our experiments. Use of Fisher's exact test between the hypothetical distribution of glomeruli and the observed distribution revealed a significant difference between the two distributions $(P=0.032)$. When we tested only the two major glomerular clusters (T1 and T3), the difference between the expected and the observed distribution had an even higher significance level $(P=0.0076)$. The projection patterns of sensilla in $S$. basiconica-enriched zones of the antenna, therefore, further indicate that axons of ORNs housed in S. basiconica preferentially, although not exclusively, project to the T3 cluster of olfactory glomeruli in the AL of worker bees.

Number of glomeruli innervated by mALT projection neurons is reduced in honeybee drones

We have shown that ORNs from $S$. basiconica preferentially project to the T3 glomerular cluster in honeybee workers. This glomerular cluster is however reduced in honeybee drones (Nishino et al. 2009) and is associated with the mALT in honeybee workers (Kirschner et al. 2006). Thus, we assumed that the mALT should also be reduced in drones. Anterograde fluorescent staining of the output tracts of the AL did not reveal any obvious differences in tract diameters between the mALT and 1ALT in confocal images (Fig. 6a). In drones, as in workers, mALT PNs project via the MBs to the LH, whereas lALT PNs project first to the LH and then to the MBs (Fig. 6a). One preparation with a mass-staining of ORN axons in drones was used to reconstruct all glomeruli of the AL (Fig. 6c). This revealed a total of 109 glomeruli (107 glomeruli were counted in a second mass-staining). Specific staining of the mALT was used to determine the number of mALT glomeruli. One backfill of the mALT PNs (Fig. 6b) was reconstructed (Fig. 6d) and revealed a total of 45 glomeruli innervated by mALT PNs. In the remaining successful mALT labelings, a similar or even smaller number of glomeruli was counted (numbers from all successful mALT stainings: specimen 1: 39, specimen 2: 45, specimen 3: 45, specimen 4: 39, specimen 5: 43, $n=5$ ). For further calculations, we used the highest number of labeled glomeruli, as some glomeruli might not have been stained by retrogradely labeling the PN arborizations in the AL. In honeybee workers, 920 PNs innervate $\sim 161$ glomeruli (Rybak 2012), resulting in a hypothetical ratio 
Fig. 4 Confocal image stacks from two antennal lobes after staining of Sensilla basiconicarich regions of the antenna (a-f) and image stacks of a singlesensillum staining of a S. basiconicum (g-i). Stained glomeruli (asterisks). a, d, g Complete stacks of antennal lobes with the antennal nerves $(A N)$ and olfactory receptor neuron (ORN) arborizations in single glomeruli can hardly be identified in the complete image stacks. Bar $100 \mu \mathrm{m}$. b, e, h Substacks of the antennal lobes with identifiable ORN innervation in individual glomeruli in the $\mathrm{T} 1$ glomerular cluster. c, f, i Substacks of the antennal lobe with identifiable ORN innervation in individual glomeruli in the $\mathrm{T} 3$ glomerular cluster region. Bar $100 \mu \mathrm{m}$
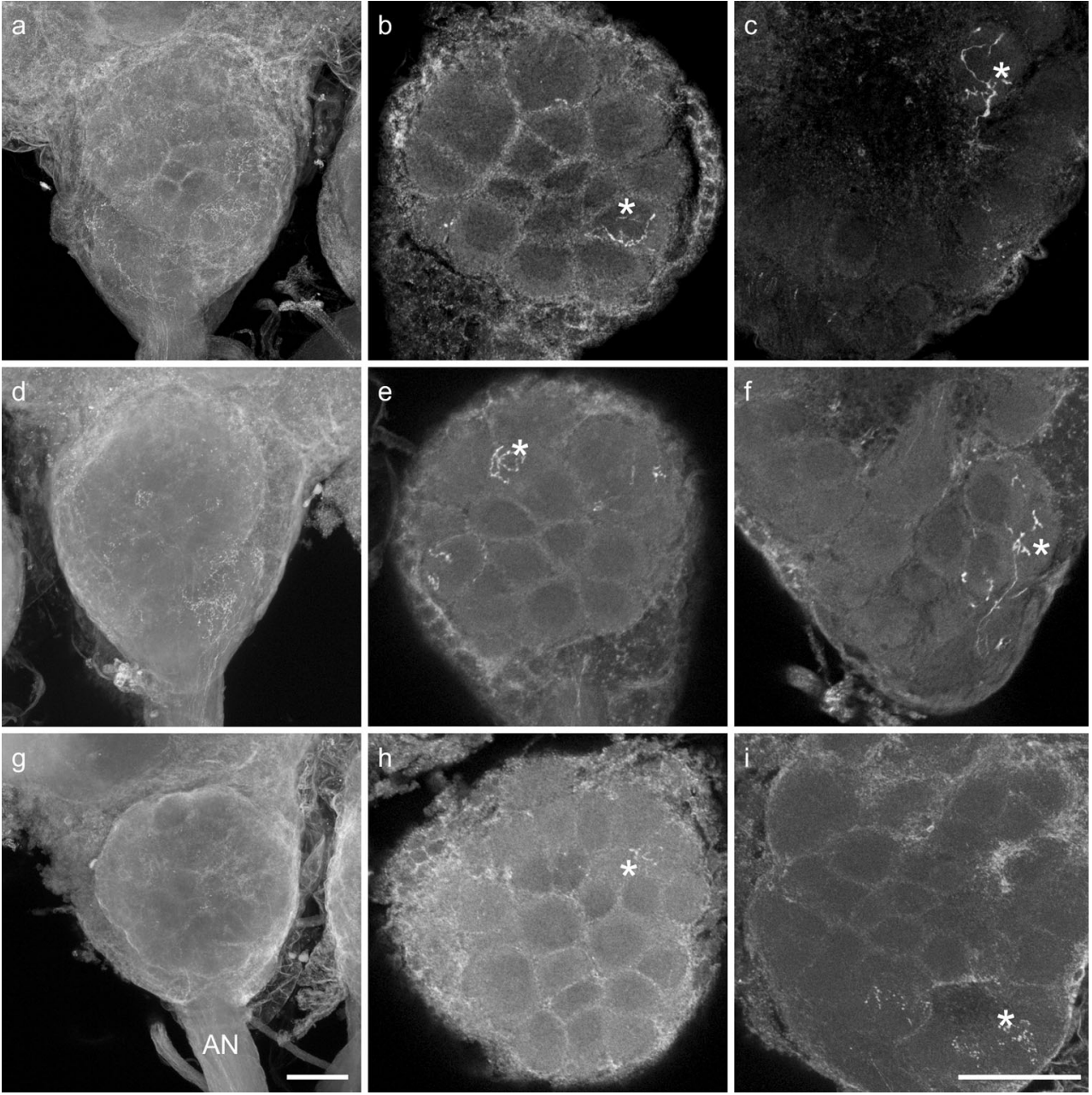

of 5-6 PNs per glomerulus. No data on the number of PNs in drones are available but by assuming multiple PNs per glomerulus and by considering the fact that only one PN needs to be stained to identify a glomerulus, we assumed that the highest numbers of glomeruli counted in our results were representative of the total number of mALT innervated glomeruli. Subtraction of the average number of maximally counted mALT-associated glomeruli (45) from the total number of 109 glomeruli revealed an estimated number of 64 1ALT-associated glomeruli (numbers in Fig. 7). Compared with the situation in females $(\sim 161$ glomeruli in total; Kirschner et al. 2006), this indicates that most of the glomeruli missing in drones can be assigned to the mALT hemilobe of the AL. In total, $24 \%$ of the 1ALT glomeruli and $42 \%$ of the mALT glomeruli were absent in drones compared with workers. Whereas the mALT to ALT ratio of the AL in females is roughly $1: 1$, only $\sim 39 \%$ of all AL glomeruli in drones were associated with the mALT compared with $\sim 61 \%$ glomeruli associated with the 1ALT. The combination of our present results with the data from Kirschner et al. (2006) indicates that ORNs from $S$. basiconica in female worker bees preferentially innervate the T3 cluster of glomeruli and that this mALT-innervated cluster of glomeruli is reduced in drones (Fig. 7).

\section{Discussion}

This study shows that axons of ORNs from S. basiconica, which are absent on drone antennae, mainly project into the T3 cluster of glomeruli in the AL of worker bees (Fig. 7). The T3 cluster of glomeruli has been demonstrated to be reduced in drones (Nishino et al. 2009) and our results reveal that mALT PNs (which, in the female $\mathrm{AL}$, receive input from the $\mathrm{T} 3$ cluster) innervate fewer glomeruli in drones compared with workers. This indicates that information from the $S$. basiconica is associated with the mALT pathway in females and further suggests that this part of the mALT pathway is reduced in drones compared with workers. A similar reduction of the mALTassociated portion of AL glomeruli has been shown in males of the ant Camponotus floridanus (Zube and Rössler 2008). 


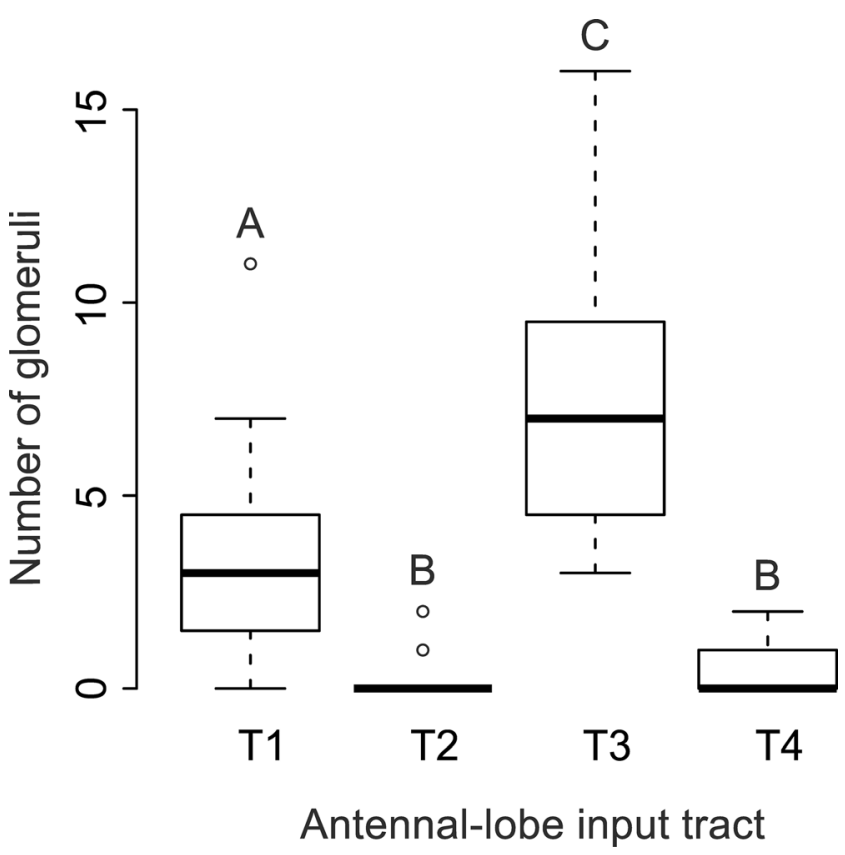

Fig. 5 Numbers of glomeruli containing axonal projections from selective staining of ORNs in multiple Sensilla basiconica. Antennal lobe input tract $\mathrm{T} 3$ glomeruli were stained significantly more often compared with $\mathrm{T} 1$ glomeruli and $\mathrm{T} 1$ glomeruli were stained more often than $\mathrm{T} 2$ and T4 glomeruli (Friedman ANOVA, post-hoc Wilcoxon rank sum test with Bonferroni correction for multiple comparisons, $n=15, A-C$ significant differences between clusters)

General features and sorting of axonal projections of ORNs

The trajectories of individual axons in the mass-stainings indicate that ORN axons are guided to their glomerular targets via a two-step sorting process, one at the segregation into T1$\mathrm{T} 4$ at the entrance of the antennal nerve and a second one at the end of the tracts, just before the axons diverge to their glomerular targets. We show that ORN axons from the various antennal segments run in parallel along the antennal nerve until they reach the AL entrance. This is analogous to the ORN axon-sorting zone in the moth Manduca sexta (Rössler et al. 1999). After the initial rearrangements at the AL entrance, however, axonal projections in the honeybee proceed again in parallel along the tracts (T1-T4) until they reach the glomerular clusters. At these glomerular clusters, ORN axons appear to be rearranged in another crossing pattern until they terminate in distinct layers within individual glomeruli. This indicates that the organization in the four olfactory sensory tracts in the honeybee involves a more complex sorting process compared with the single sorting zone at the AL entrance shown in moths (Rössler et al. 1999). This process requires further clarification in future developmental studies.

Within individual glomeruli, ORN axons of sensilla from the distal and proximal segments of the antenna terminate in different layers (Fig. 2). These projection patterns indicate a change from the topographic position of sensilla on the antenna to the spatial organization of ORN target fields within single AL glomeruli. In contrast to the ORN projections, PN dendrites might arborize throughout the entire glomerulus (Fig. 2c, d; see also Müller et al. 2002; Krofczik et al. 2008) indicating that individual PNs receive convergent input from ORNs from widely separated topographical locations that innervate the same glomerulus.

\section{Glomerular targets of ORN axons from S. trichoidea}

Selective mass-staining of $S$. trichoidea labels bundles of ORN axons projecting to the AL (Fig. 3). The trajectories of ORN axons can be followed along three tracts (T1, T2, T3; Fig. 3). In none of the 13 preparations have we found staining of T4 glomeruli (Fig. 3d). Interestingly, despite a wide distribution of axons in almost all glomeruli of the AL, slightly brighter staining has been detected in T3 glomeruli compared with $\mathrm{T} 1$ glomeruli. Whether this is attributable to a higher density and/or brighter labeling of individual axons cannot be resolved with our method. ORN axons from $S$. placodea have also been shown to project into all four glomerular clusters in the honeybee (Kelber et al. 2006). For a more detailed analysis, single fills of $S$. trichoidea ORNs would be necessary. Our staining demonstrates that ORN projections from $S$. trichoidea from only one antennal segment are widely distributed across three glomerular clusters (T1-T3) that contain $>95 \%$ of all AL glomeruli in the honeybee (Kirschner et al. 2006). Assuming that this is true for all antennal segments (we found similar results for segments $3,5,6,7$ and 8), this implies a high redundancy of the antennal segments within individual glomeruli. This ensures that the antenna efficiently captures sufficient odor molecules for odor identification but rather excludes a topographical resolution of odor reception (e.g., ORNs at the proximal or distal part of the antenna). In contrast to ORNs, PNs have dendritic arborizations across entire glomeruli (Fig. 2c, d). Whether the position of ORN projections in a distinct layer of the glomerulus (and its position on the antenna) in the honeybee has any physiological impact on PN responses, however, needs to be investigated in physiological recordings.

\section{Projection patterns of ORN axons from S. basiconica}

Mass-staining techniques of hair-like sensilla on entire antennal segments have not allowed us to resolve glomerular cluster preferences of $S$. basiconica-associated ORN axons, as even the $S$. basiconica-rich segment 9 contains over $10 \times$ more S. trichoidea than S. basiconica (Esslen and Kaissling 1976). To increase the proportion of $S$. basiconica-associated ORNs, we selectively stained hair-like sensilla in the distal regions of individual antennal segments that show a ring-like arrangement of $S$. basiconica (Fig. 1). These 
Fig. 6 a Z-projection of a massstaining of antennal lobe $(A L)$ projection neurons in the honeybee drone; the AL, the medial and the lateral AL tract ( $m A L T, l A L T)$ and arborizations in the mushroom bodies $(M B)$ and the lateral horn $(L H)$ are visible. Bar $200 \mu \mathrm{m}$. b Z-projection of mALT staining in a honeybee drone. The axons, cell bodies $(C B)$ and dendritic glomerular $(G L)$ innervation are visible. Bar $100 \mu \mathrm{m}$. c Three-dimensional reconstruction of a drone $\mathrm{AL}$ with the antennal nerve $(A N)$ after staining of all olfactory receptor neuron axons. Two macroglomeruli $(M G L)$ are indicated. $\mathbf{d}$ Reconstruction of the mALT proportion of glomeruli and two macroglomeruli ( $M G L)$ within the $\mathrm{T} 1$ glomerular cluster (TI) as landmarks in a drone AL. The two 1ALT-associated MGL are shown in green, whereas mALT glomeruli in the T3 glomerular cluster (T3) are shown in shades of magenta. Bar $100 \mu \mathrm{m}$
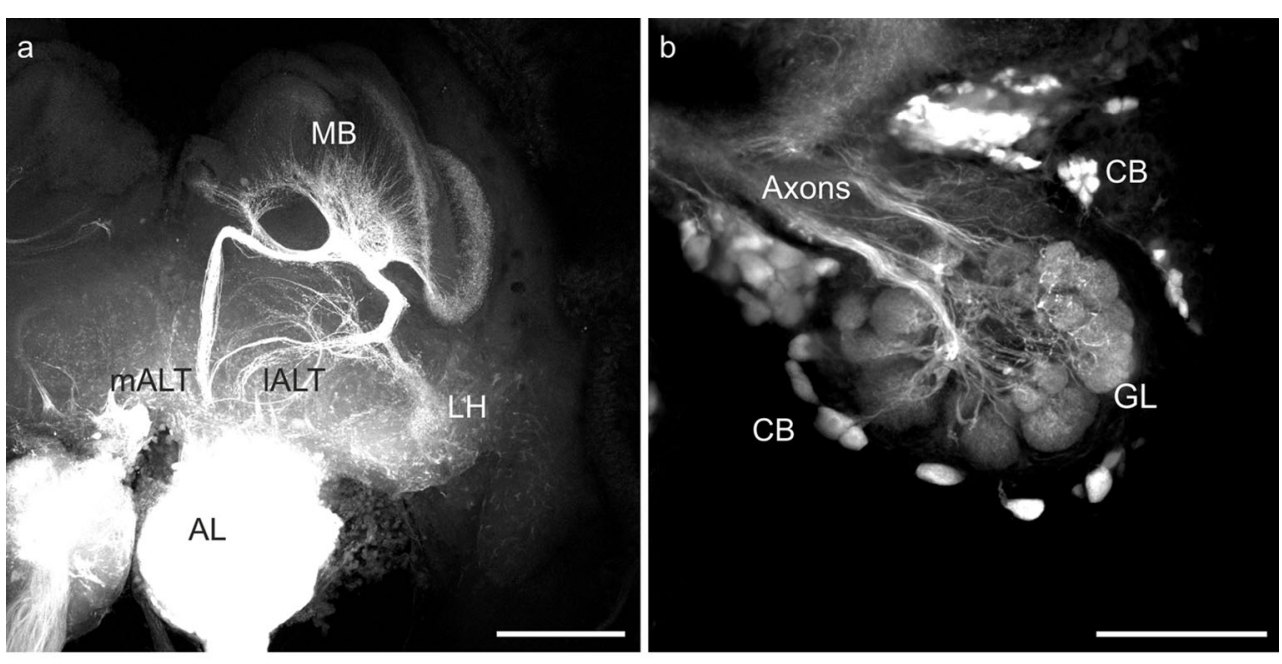

C

d

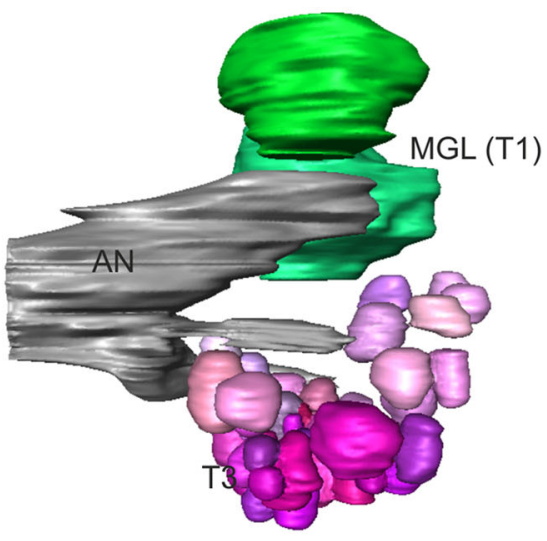

experiments revealed that ORN axons from these sensilla terminate to a significantly higher proportion in glomeruli of the T3 cluster of AL glomeruli (Figs. 5, 7). In these staining experiments, we have preferentially aimed to cut $S$. basiconica but because of the small size of these sensilla in the honeybee, staining of $S$. trichoidea, in most cases, cannot be completely excluded. Therefore, we used a statistical method to analyze cluster preference in these selective stainings. Furthermore, the approved staining (by visual inspection) of a single $S$. basiconicum has shown that nine of the T3 glomeruli and only one T1 glomerulus are innervated thereby supporting the multiple sensilla staining results. The single $S$. basiconicum stained in our experiments comprised 10 ORNs. This is in agreement with results from histological investigations showing that $S$. basiconica in the honeybee contain 8-12 ORN dendrites (Nishino et al. 2009). In other Hymenoptera, ORN numbers in $S$. basiconica have been demonstrated to range between 30-40 ORNs in wasps (Lacher 1964) and 3-53 ORNs in ants (Kelber et al. 2010). Until now, the notion that $S$. basiconica are indeed olfactory sensilla is mainly based on morphological criteria. Whereas the morphological evidence is strong, however, clear physiological evidence is still lacking. Recordings from S. basiconica have only been mentioned as unpublished results in Akers and Getz (1993). Our anterograde staining of the axonal projections of ORNs housed in S. basiconica has revealed innervations of relatively high numbers of preferentially $\mathrm{T} 3$ glomeruli in the AL. This adds further evidence to the assertion that $S$. basiconica are olfactory sensilla (Slifer and Sekhon 1961; Lacher 1964). Our results suggest that axons of S. basiconica-associated ORNs preferentially project to glomeruli of the T3 cluster and only partially to glomeruli of the T1 cluster.

Differences between honeybee drones and workers and potential functional consequences

Interestingly, S. basiconica are present in honeybee workers and queens but not in drones (Esslen and Kaissling 1976). The absence of $S$. basiconica in males seems to be a typical feature in Hymenoptera including the honeybee, bumblebees, solitary bees and ants (Ågren 1977; 1978; Wcislo 1995; Ågren and Hallberg 1996; Nakanishi et al. 2009, 2010; Mysore et al. 2010; Nishikawa et al. 2012; Streinzer et al. 
Fig. 7 Representations of a honeybee worker $(l e f t)$ and drone (right) antennal lobe (AL). Olfactory receptor neuron axons housed in S. basiconica are only present in workers and project via the antennal nerve mainly to the $\mathrm{T} 3$ glomerular cluster (T3) in the $\mathrm{AL}$ (light blue projections). The $\mathrm{T} 1$ glomerular cluster $(T 1)$ is drawn in shades of green, the $\mathrm{T} 2$ in shades of yellow (T2) and the T4 cluster in shades of blue (T4). The medial AL tract ( $m A L T$ ) innervates mainly $\mathrm{T} 3$ glomeruli and the lateral $\mathrm{AL}$ tract (IALT) mainly T1 glomeruli. The T3 glomerular cluster and the mALT output tract are reduced in drones. The estimated numbers of glomeruli innervated by mALT and IALT projection neurons and the total numbers of glomeruli in the female (from Kirschner et al. 2006) and male AL are indicated (ORNs olfactory receptor neurons)

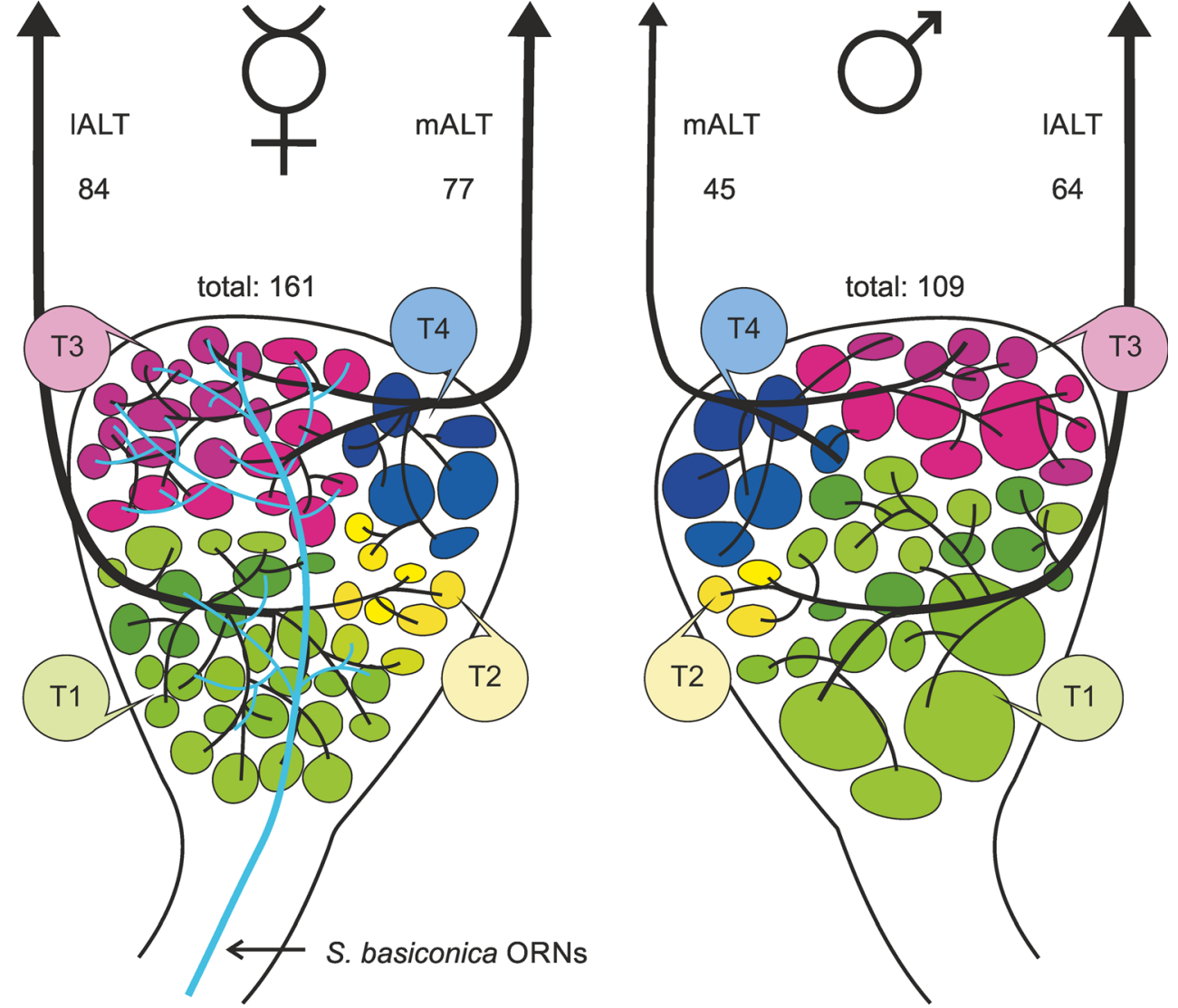

2013). Wcislo (1995) argued that drones have reduced the number of a certain type of olfactory sensilla to increase their capabilities for pheromone detection and thereby to increase their probability for encountering a queen faster than other drones. The finding that drones have extremely high numbers of pore plates (S. placodea), which also house the pheromonespecific sensilla (Kaissling and Renner 1968), supports this view. Three of the enlarged glomeruli (macroglomeruli) are located in the T1 cluster of glomeruli (Nishino et al. 2009) and calcium-imaging studies have shown that components of the queen mandibular pheromone are processed in one of these enlarged glomeruli (Sandoz 2006). The processing of pheromone information in macroglomeruli is well known from sex pheromone communication in various species of moths (e.g., Christensen and Hildebrand 1987; Anton and Hansson 1994; Greiner et al. 2004) and has also been shown for an enlarged glomerulus which processes components of the trail pheromone in large leaf-cutting ant workers (Kleineidam et al. 2005; Kelber et al 2009; Kuebler et al 2010). The reduced number of $S$. trichoidea and the complete absence of $S$. basiconica and the associated neuronal pathway in the AL of drones might allow higher capacities for queen-pheromone-specific sensilla and a larger sex-pheromone-processing neuronal circuitry in the AL (Fig. 7).
Female-specific olfactory subsystems and their possible function

We have been able to demonstrate that the reduction of glomeruli in the AL of honeybee drones is higher in the mALTcompared to the 1ALT-innervated hemilobe of the AL. ORN axons in the glomeruli of the T3 cluster are innervated by mALT PNs in females (Kirschner et al. 2006). This cluster is reduced in drones, whereas the T1 cluster is less reduced compared with that in the female AL. As drones lack $S$. basiconica, the reduction of the mALT-associated parts of the T3 glomeruli is likely to be related to the absence of ORNs from the $S$. basiconica. As three enlarged glomeruli (macroglomeruli, as indicated in Fig. 7) are present in the T1 cluster in drones (Sandoz 2006; Nishino et al. 2009), the slight reduction in the number of $\mathrm{T} 1$ glomeruli in drones might favor the macroglomeruli.

Several physiological studies, so far, have shown that no part of the honeybee AL and therefore neither the MALT or the IALT, is selectively specialized for either only social or floral odorants (Abel et al. 2001; Brill et al. 2013; Carcaud et al. 2012; Galizia et al. 2012; Krofczik et al. 2008; Müller et al. 2002; Rössler and Brill 2013; Yamagata et al. 2009). However, as honeybees are exposed to an enormous odor space in their natural environments, more odorants, in 
particular social (colony) cues and pheromones, remain to be tested in more detail and might give further indications concerning selective physiological properties and the molecular receptive range of $S$. basiconica ORNs. The ants Camponotus floridanus (Zube and Rössler 2008), Camponotus japonicus (Nishikawa et al. 2012) and A. vollenweideri (Kelber et al. 2010) have similarly been demonstrated to have a reduced number of glomeruli in males compared with females. Kelber et al. (2010) investigated projection patterns of $S$. trichoidea and S. basiconica ORNs in A. vollenweideri. Here, the ants possess six AL glomerular clusters (T1-T6) and S. trichoidea ORNs have been shown to project to all of them. Up to five different glomerular clusters have been found to be innervated by ORNs from a single S. trichoideum. In contrast, S. basiconica ORNs only projected to the T6 cluster (Kelber et al. 2010). In honeybee workers, we were able to reveal that $S$. basiconica preferentially project into the $\mathrm{T} 3$ cluster. $C$. japonicus ALs comprise seven glomerular clusters (T1-T7) and the glomerular cluster T6 is also worker-specific, similar to the T6 cluster in A. vollenweideri (Kelber et al. 2010; Nishikawa et al. 2012). Furthermore, here the T6 output neurons were demonstrated to project to specific subregions within the MBs and the $\mathrm{LH}$ and the authors speculate that the input to these glomeruli origins from $S$. basiconica-associated ORNs (Nishikawa et al. 2012). Nishikawa et al. (2012) argued that these brain regions are likely to be involved in social tasks in C. japonicus, as drones do not need to fulfil extensive social duties within the colony.

Another important aspect is that in several solitary bee species, the males also lack S. basiconica (Agren 1977, 1978; Ågren and Hallberg 1996; Galvani 2012; Streinzer et al. 2013). In parasitoid wasps, cuticular hydrocarbon profiles have been shown to vary between closely related species, between sexes and according to the developmental environment (Khidr et al. 2013). Better detection abilities for cuticular hydrocarbons could serve females in kin detection and thus help them to avoid insemination by related males or even by males from other species. Another major distinction between drones and workers in both ants and social bees is that drones do not forage. This implies the involvement of S. basiconica in the detection of floral odorants. These studies show that various hymenopteran species possess female-specific olfactory subsystems consisting in specific sensilla, their respective ORNs and downstream odor-processing brain structures. The separation of these subsystems from the sexual isomorphic structures can be more (A. vollenweideri, C. japonicus) or less (A. mellifera) pronounced. Thus, variations in olfactory tasks and structures between males and females might differ across species.

Based on these differences described so far with regard to behaviors between male and female honeybees, we conclude that the $S$. basiconica might equally well be specialized for flower odorant detection or the detection of social olfactory cues or might even be generalistic.

\section{Concluding remarks}

Honeybee workers possess a specific olfactory subsystem comprising $S$. basiconica, parts of the T3 cluster of glomeruli and a significant proportion of mALT PNs. Drones completely lack this olfactory subsystem (Fig. 7) and, additionally, have far fewer S. trichoidea, all features that might favor a more elaborated queen-pheromone-processing system and the associated higher numbers of $S$. placodea present in drones (Brockmann et al. 2006; Kaissling and Renner 1968; Sandoz 2006). At the behavioral level, drones, therefore, are likely to have more limited odor discrimination and recognition abilities compared with females. This limitation is likely to be associated with social (colony) odors and/or floral odors. The adaptation in drones for improved queen-pheromone detection including high numbers of $S$. placodea and pheromone-processing macroglomeruli is likely to increase mating probabilities. Honeybee workers, in contrast, are exposed to high selective pressure to identify and locate correctly a wide variety of odorants, including floral odorant mixtures, a large variety of pheromones and colony (social) cues. The different types of sensilla and the associated olfactory subsystems of glomeruli and output tracts in the AL appear to be well adapted for these tasks.

Acknowledgments We thank Cornelia Grübel for expert help with tract staining and confocal microscopy, Dirk Ahrens for beekeeping and Johannes Spaethe for fruitful discussions and help with statistics.

Open Access This article is distributed under the terms of the Creative Commons Attribution License which permits any use, distribution, and reproduction in any medium, provided the original author(s) and the source are credited.

\section{References}

Abel R, Rybak J, Menzel R (2001) Structure and response patterns of olfactory interneurons in the honeybee, Apis mellifera. J Comp Neurol 437:363-383

Ache BW, Young JM (2005) Olfaction: diverse species, conserved principles. Neuron 48:417-430

Ågren L (1977) Flagellar sensilla of some colletidae (Hymenoptera: Apoidea). Int J Insect Morphol Embryol 6:137-146

Ågren L (1978) Flagellar sensilla of two species of Andrena (Hymenoptera: Andrenidae). Int J Insect Morphol Embryol 7:73-79

Ågren L, Hallberg E (1996) Flagellar sensilla of bumble bee males (Hymenopera, Apidae, Bombus). Apidologie 27:433-444

Ai H, Nishino H, Itoh T (2007) Topographic organization of sensory afferents of Johnston's organ in the honeybee brain. J Comp Neurol 502:1030-1046 
Akers RP, Getz WM (1993) Response of olfactory receptor neurons in honeybees to odorants and their binary mixtures. J Comp Physiol A 173:169-185

Anton S, Hansson BS (1994) Central processing of sex pheromone, host odour, and oviposition deterrent information by interneurons in the antennal lobe of female Spodoptera littoralis (Lepidoptera: Noctuidae). J Comp Neurol 350:199-214

Anton S, Homberg U (1999) Antennal lobe structure. In: Hansson B (ed) Insect olfaction. Springer, Berlin, pp 97-124

Arnold G, Masson C, Budharugsa S (1985) Comparative study of the antennal lobes and their afferent pathway in the worker bee and the drone (Apis mellifera). Cell Tissue Res 242:2379-2383

Brill MF, Rosenbaum T, Reus I, Kleineidam CJ, Nawrot MP, Rossler W (2013) Parallel processing via a dual olfactory pathway in the honeybee. J Neurosci 33:2443-2456

Brockmann A, Brückner D (1995) Projection pattern of poreplate sensory neurones in honey bee worker, Apis mellifera L. (Hymenoptera: Apidae). Int J Insect Morphol Embryol 24:405-411

Brockmann A, Dietz D, Spaethe J, Tautz J (2006) Beyond 9-ODA: sex pheromone communication in the European honey bee Apis mellifera L. J Chem Ecol 32:657-667

Carcaud J, Hill T, Giurfa M, Sandoz J-C (2012) Differential coding by two olfactory subsystems in the honeybee brain. J Neurophysiol 108:1106-1121

Cervantes-Sandoval I, Martin-Peña A, Berry JA, Davis RL (2013) System-like consolidation of olfactory memories in Drosophila. J Neurosci 33:9846-9854

Christensen TA, Hildebrand JG (1987) Male-specific, sex pheromoneselective projection neurons in the antennal lobes of the moth, Manduca sexta. J Comp Physiol A 160:553-569

Dacks AM, Nighorn AJ (2011) The organization of the antennal lobe correlates not only with phylogenetic relationship, but also life history: a basal hymenopteran as exemplar. Chem Senses 36:209220

Davis RL (2005) Olfactory memory formation in Drosophila: from molecular to systems neuroscience. Annu Rev Neurosci 28:275-302

Esslen J, Kaissling K (1976) Zahl und Verteilung antennaler Sensillen bei der Honigbiene Apis mellifera L. Zoomorphology 83:227-251

Flanagan D, Mercer A (1989) An atlas and 3-D reconstruction of the antennal lobes in the worker honey bee, Apis mellifera L. (Hymenoptera: Apidae). Int J Insect Morphol Embryol 18:145-159

Galizia CG, Franke T, Menzel R, Sandoz J-C (2012) Optical imaging of concealed brain activity using a gold mirror in honeybees. J Insect Physiol 58:743-749

Galizia CG, McIlwrath SL, Menzel R (1999) A digital three-dimensional atlas of the honeybee antennal lobe based on optical sections acquired by confocal microscopy. Cell Tissue Res 295:383-394

Galizia CG, Rössler W (2010) Parallel olfactory systems in insects: anatomy and function. Annu Rev Entomol 55:399-420

Galvani G (2012) Distribution and morphometric studies of flagellar sensilla in Emphorini bees (Hymenoptera, Apoidea). Micron 43: 673-687

Gary NE (1962) Chemical mating attractants in the queen honey bee. Science 136:773-774

Gerber B, Tanimoto H, Heisenberg M (2004) An engram found? Evaluating the evidence from fruit flies. Curr Opin Neurobiol 14: 737-744

Getz WM, Akers RP (1993) Olfactory response characteristics and tuning structure of placodes in the honey bee Apis mellifera L. Apidologie 24:195-217

Giurfa M (2007) Behavioral and neural analysis of associative learning in the honeybee: a taste from the magic well. J Comp Physiol A 193: 801-824

Greiner B, Gadenne C, Anton S (2004) Three-dimensional antennal lobe atlas of the male moth, Agrotis ipsilon: a tool to study structurefunction correlation. J Comp Neurol 475:202-210
Groh C, Rössler W (2008) Caste-specific postembryonic development of primary and secondary olfactory centers in the female honeybee brain. Arthropod Struct Dev 37:459-468

Hildebrand JG, Shepherd GM (1997) Mechanisms of olfactory discrimination: converging evidence for common principles across phyla. Annu Rev Neurosci 20:595-631

Hourcade B, Muenz TS, Sandoz J-C, Rössler W, Devaud J-M (2010) Long-term memory leads to synaptic reorganization in the mushroom bodies: a memory trace in the insect brain? J Neurosci 30:6461-6465

Ito K, Shinomiya K, Ito M, Armstrong JD, Boyan G, Hartenstein V, Harzsch S, Heisenberg M, Homberg U, Jenett A, Keshishian H, Restifo LL, Rössler W, Simpson JH, Strausfeld NJ, Strauss R, Vosshall LB, Insect Brain Name Working Group (2014) A systematic nomenclature for the insect brain. Neuron 81:755-765

Kaissling KE, Renner M (1968) Antennal receptors for queen substance and scent gland odour in honeybees. Z Vergl Physiol 59:357-361

Kelber C, Rössler W, Kleineidam CJ (2006) Multiple olfactory receptor neurons and their axonal projections in the antennal lobe of the honeybee Apis mellifera. J Comp Neurol 496:395-405

Kelber C, Rössler W, Roces F, Kleineidam CJ (2009) The antennal lobes of fungus-growing ants (Attini): neuroanatomical traits and evolutionary trends. Brain Behav Evol 73:273-284

Kelber C, Rössler W, Kleineidam CJ (2010) Phenotypic plasticity in number of glomeruli and sensory innervation of the antennal lobe in leafcutting ant workers (A. vollenweideri). Dev Neurobiol 70:222-234

Khidr SK, Linforth RST, Hardy ICW (2013) Genetic and environmental influences on the cuticular hydrocarbon profiles of Goniozus wasps. Entomol Exp Appl 147:175-185

Kirschner S, Kleineidam CJ, Zube C, Rybak J, Grünewald B, Rössler W (2006) Dual olfactory pathway in the honeybee, Apis mellifera. J Comp Neurol 499:933-952

Kleineidam CJ, Obermayer M, Halbich W, Rössler W (2005) A macroglomerulus in the antennal lobe of leaf-cutting ant workers and its possible functional significance. Chem Senses 30:383-392

Krofczik S, Menzel R, Nawrot MP (2008) Rapid odor processing in the honeybee antennal lobe network. Front Comput Neurosci 2:9

Kuebler LS, Kelber C, Kleineidam CJ (2010) Distinct antennal lobe phenotypes in the leaf-cutting ant (Atta vollenweideri). J Comp Neurol 518:352-365

Lacher V (1964) Elektrophysiologische Untersuchungen an einzelnen Rezeptoren für Geruch, Kohlendioxyd, Luftfeuchtigkeit und Tempratur auf den Antennen der Arbeitsbiene und der Drohne (Apis mellifica L.). Z Vergl Physiol 48:587-623

Le Conte Y, Hefetz A (2008) Primer pheromones in social Hymenoptera. Annu Rev Entomol 53:523-542

Maronde U (1991) Common projection areas of antennal and visual pathways in the honeybee brain, Apis mellifera. J Comp Neurol 309:328-340

Martin JP, Beyerlein A, Dacks AM, Reisenman CE, Riffell JA, Lei H, Hildebrand JG (2011) The neurobiology of insect olfaction: sensory processing in a comparative context. Prog Neurobiol 95:1-21

Mobbs PG (1982) The brain of the honeybee Apis mellifera. I. The connections and spatial organization of the mushroom bodies. Proc R Soc Lond [Biol] 298:309-354

Müller D, Abel R, Brandt R, Zöckler M, Menzel R (2002) Differential parallel processing of olfactory information in the honeybee, Apis mellifera L. J Comp Physiol A 188:359-370

Mysore K, Shyamala BV, Rodrigues V (2010) Morphological and developmental analysis of peripheral antennal chemosensory sensilla and central olfactory glomeruli in worker castes of Camponotus compressus (Fabricius, 1787). Arthropod Struct Dev 39:310-321

Nakanishi A, Nishino H, Watanabe H, Yokohari F, Nishikawa M (2009) Sex-specific antennal sensory system in the ant Camponotus japonicus: structure and distribution of sensilla on the flagellum. Cell Tissue Res 338:79-97 
Nakanishi A, Nishino H, Watanabe H, Yokohari F, Nishikawa M (2010) Sex-specific antennal sensory system in the ant Camponotus japonicus: glomerular organizations of antennal lobes. J Comp Neurol 518:2186-2201

Nishikawa M, Watanabe H, Yokohari F (2012) Higher brain centers for social tasks in worker ants, Camponotus japonicus. J Comp Neurol 520:1584-1598

Nishino H, Nishikawa M, Mizunami M, Yokohari F (2009) Functional and topographic segregation of glomeruli revealed by local staining of antennal sensory neurons in the honeybee Apis mellifera. J Comp Neurol 515:161-180

Palmer MJ, Moffat C, Saranzewa N, Harvey J, Wright GA, Connolly CN (2013) Cholinergic pesticides cause mushroom body neuronal inactivation in honeybees. Nat Commun 4:1634

Rössler W, Brill MF (2013) Parallel processing in the honeybee olfactory pathway: structure, function, and evolution. J Comp Physiol A 199: 981-996

Rössler W, Zube C (2011) Dual olfactory pathway in Hymenoptera: evolutionary insights from comparative studies. Arthropod Struct Dev 40:349-357

Rössler W, Oland LA, Higgins MR, Hildebrand JG, Tolbert LP (1999) Development of a glia-rich axon-sorting zone in the olfactory pathway of the moth Manduca sexta. J Neurosci 19:9865-9877

Rybak J (2012) The digital honey bee brain atlas. In: Eisenhardt D, Giurfa M, Galizia CG (eds) Honeybee neurobiology and behaviora tribute to Randolf Menzel. Springer, The Netherlands, pp $125-140$

Sandoz J-C (2006) Odour-evoked responses to queen pheromone components and to plant odours using optical imaging in the antennal lobe of the honey bee drone Apis mellifera L. J Exp Biol 209:35873598

Slessor KN, Winston ML, Le Conte Y (2005) Pheromone communication in the honeybee (Apis mellifera L.). J Chem Ecol 31:2731-2745

Slifer EH, Sekhon SS (1961) Fine structure of the sense organs on the antennal flagellum of the honey bee, Apis mellifera Linnaeus. J Morphol 109:351-381

Streinzer M, Kelber C, Pfabigan S, Kleineidam CJ, Spaethe J (2013) Sexual dimorphism in the olfactory system of a solitary and a eusocial bee species. J Comp Neurol 521:2742-2755

Touhara K, Vosshall LB (2009) Sensing odorants and pheromones with chemosensory receptors. Annu Rev Physiol 71:307-332

Wanner KW, Nichols AS, Walden KKO, Brockmann A, Luetje CW, Robertson HM (2007) A honey bee odorant receptor for the queen substance 9-oxo-2-decenoic acid. Proc Natl Acad Sci U S A 104: $14383-14388$

Wcislo WT (1995) Sensilla numbers and antennal morphology of parasitic and non-parasitic bees (Hymenoptera: Apoidea). Int J Insect Morphol Embryol 24:63-81

Yamagata N, Schmuker M, Szyszka P, Mizunami M, Menzel R (2009) Differential odor processing in two olfactory pathways in the honeybee. Front Syst Neurosci 3:16

Zube C, Rössler W (2008) Caste- and sex-specific adaptations within the olfactory pathway in the brain of the ant Camponotus floridanus. Arthropod Struct Dev 37:469-479

Zube C, Kleineidam CJ, Kirschner S, Neef J, Rössler W (2008) Organization of the olfactory pathway and odor processing in the antennal lobe of the ant Camponotus floridanus. J Comp Neurol 506:425-441 